\title{
Information and knowledge management in online rich presence services
}

\author{
Casey K. Fung • Patrick C. K. Hung
}

Published online: 27 July 2013

(C) Springer Science+Business Media New York 2013

\section{About this issue}

Nowadays many information technology services are moving towards online via ubiquitous networks, computing and information at unlimited speed anytime and anywhere with different kinds of technical platforms, especially in Web 2.0. In general, presence information expresses user willingness and ability to communicate with other users across a set of services for real-time communications. Web 2.0 uses the Web as a platform for embracing the interconnectivity and interactivity of Web-delivered content among different online users and communities, which have recently been assumed by the technology of services computing such as Web services. Web services are based on a set of XML standards such as Universal Description, Discovery and Integration (UDDI), Web Services Description Language (WSDL), Simple Object Access Protocol (SOAP), Business Process Execution Language for Web Services (BPEL4WS), and etc. The rapid development of Web 2.0 for massive social network collaboration facilitates the "rich presence services" to expose information and knowledge gathered through online social networks which assists intelligent decision making and analysis.

Rich presence is described as an enhanced form of presence awareness in which a service can observe other online service/user attributes, such as personal identifiable information, location, time, behavior, movement, type-of-software,

\author{
C. K. Fung \\ Boeing Research and Technology, Seattle, WA, USA \\ e-mail: casey.k.fung@boeing.com \\ P. C. K. Hung \\ University of Aizu, Aizu, Japan \\ P. C. K. Hung $(\bowtie)$ \\ University of Ontario Institute of Technology (UOIT), \\ Oshawa, Canada \\ e-mail: patrick.hung@uoit.ca
}

type-of-device, and type-of-network. The use of Web 2.0 technologies may generate both user-generated content as well as contextual information about users, for example, their current location and availability. Rich presence also refers to the aggregation of this information that makes presence attributed to consenting services available for use by other services/users. This result generates a consolidation of virtual, physical, and online services into a single richpresence platform based on content-based publish/subscribe services for efficient filtering and dissemination of presence information. Online rich presence services divulge only the availability of other services.

In this context, knowledge management comprises a range of strategies and practices used in an online rich presence service to create, manage, coordinate, distribute, and enable adoption of information. Such information comprises knowledge, either embedded in services or embedded in business processes, focuses on organizational quality of services (QoS) objectives such as performance, and competitiveness. Therefore, this special issue aims to examine the information and knowledge management model in this emerging context for the next generation applications.

All the 8 papers in this issue will have deep research results to report the advance of online rich presence services such as information discovery, knowledge management, recommendation and collaborative filtering, services compositions, supply chain management, trust framework, grid and cloud computing. In the first paper "Feasilibility and A Case Study on Content Optimization Services on Cloud" (Wong 2013), Wong focuses on content optimization services that modify and reorganize content to reduce the size of content and enhance the performance of processing on the content. Wong presents the economics related to these services with practical considerations when these services are implemented on a cloud, which is typically perceived to be a more economical and scalable option compared to traditional dedicated servers. 
The second paper "Web 2.0 Recommendation Service by Multi-Collaborative Filtering Trust Network Algorithm" (Wei et al. 2013), Wei et al. present a Multi-Collaborative Filtering Trust Network algorithm with simulation experiments, which is an improved version of the Collaborative Filtering (CF) algorithm designed to work on the Web 2.0 platform, such as in current social networks, the required information may be either incomplete or scattered over different sources. Next the third paper "QoS-Aware MultiGranularity Service Composition" (Feng et al. 2013), Feng et al. discuss the challenge of multi-granularity service composition in order to enhance the quality of service composition by allowing services of various granularities to be available for selection. Feng et al. present a behavioral signature model in order to represent observable behavior of service composition with an illustrative case study and prototype for demonstrate and evaluate our approach.

Further, the forth paper "Optimizing Dynamic Supply Chain Formation in Supply Mesh Using CSET Model" (Yang and Fong 2013), Yang and Fong present a multiagent model called the collaborative single machine earliness/tardiness (CSET) model in a supply mesh, and the corresponding allocation schemes are experimentally studied in simulations. Yang and Fong show the experimental results based on samples from the U.S. textile industry. In addition, Yang and Fong show that a cooperative competition scheme is superior in terms of optimal allocation, which obtains maximum satisfaction for all participants. Next, the fifth paper "A Reliable Scheduling Method in Equipment Grid using Provenance Information" (Wang and Yin 2013), Wang and Yin present a fuzzy scheduling method using provenance information, in which resource dispatch probability is dynamically adjusted according to user feedback information by fuzzy variables. Wang and Yin show simulation results which confirm capability of the method to effectively reduce impacts of malicious user appreciations and increase user satisfactions.

Then the sixth paper "A Collaborative Food Safety Service Agent Architecture with Alerts and Trust" (Lai et al. 2013), Lai et al. present a Collaborative Food Safety Agent System (CFSAS) based on a scalable, flexible, and intelligent Multi-Agent Information System (MAIS) architecture for proactive aids to citizens with trust concept. Chiu et al. show an implementation with Web service technologies to integrate disparate food information resources along the food supply chain, and evaluate the approach from different stakeholders' perspectives.

The seventh paper "Improving Efficiency of Service Discovery Using Linked Data-based Service Publication" (Chen and Paik 2013), Chen and Paik present a methodology to build a global social service network based on link data principles with linked social services. Chen and Paik discuss experimental results which show that the linked social service can reduce the using threshold by enabling exploring service to service based on the global social service network. Lastly the eighth paper "Rank-Mediated Collaborative Tagging Recommendation Service Using Video-Tag Relationship Prediction" (Tung and Lee 2013) present a rankmediated collaborative tagging recommendation service that predicts the ranks of video retrieval from the shared video archive using vote-promotion algorithm.

The goal of this special issue is to crystallize the emerging rich presence service technologies and trends into positive efforts to focus on the most promising solutions in information and knowledge management. The papers provide clear proof that online rich presence service technologies are playing a more and more important and critical role in supporting business processes and applications. It is also believed that these papers will further research new best practices and directions in rich presence services.

\section{References}

Chen, W., \& Paik, I. (2013). Improving efficiency of service discovery using linked data-based service publication. Information Systems Frontiers, 15(4). doi: 10.1007/s10796-012-9381-x

Feng, Z., Peng, R., Wong, R. K., He, K., Wang, J., Hu, S., \& Li, B. (2013). QoS-aware multi-granularity service composition. Information Systems Frontiers, 15(4). doi: 10.1007/s10796-012-9378-5

Lai, W. W. K., Chiu, D. K. W., \& Feng, Z. (2013). A collaborative food safety service agent architecture with alerts and trust. Information Systems Frontiers, 15(4). doi: 10.1007/s10796-012-9382-9

Tung, W.-F., \& Lee, T.-Y. (2013). Rank-mediated collaborative tagging recommendation service using video-tag relationship prediction. Information Systems Frontiers, 15(4). doi: 10.1007/s10796-0139436-7

Wang, Y., \& Yin, J. (2013). A reliable scheduling method in equipment grid using provenance information. Information Systems Frontiers, 15(4). doi: 10.1007/s10796-013-9437-6

Wei, C., Khoury, R., \& Fong, S. (2013). Web 2.0 recommendation service by multi-collaborative filtering trust network algorithm. Information Systems Frontiers, 15(4). doi: 10.1007/s10796-0129377-6

Wong, R. K. (2013). Feasibility and a case study on content optimization services on cloud. Information Systems Frontiers, 15(4). doi: 10.1007/s10796-012-9379-4

Yang, H., \& Fong, S. (2013). Optimizing dynamic supply chain formation in supply mesh using CSET model. Information Systems Frontiers, 15(4). doi: 10.1007/s10796-012-9380-y

Casey Fung, Ph.D. is an Associate Technical Fellow of Boeing Research and Technology, Seattle. His research interest is in the areas of wireless aviation services, mobile ad hoc network systems, and service computing. $\mathrm{He}$ has been principal investigator for wireless operations for multinetwork sharing, fault tolerant distributed computing, and survivability of mobile ad hoc network system in Boeing. He was the recipient of the best research project in 2008. He has published over 25 refereed papers and is a holder of three U.S. patents on distributed computing. He has given tutorials in service computing conferences, served as special editors 
of technical journals and panel chairs of service computing conferences. He was affiliate assistant professor at University of Washington, Seattle. He obtained B.S. in Electrical Engineering from Ohio University, and $\mathrm{Ph} . \mathrm{D}$. in Computer and Information Science from Ohio State University, Columbus. Contact him at casey.k.fung@boeing.com.

Patrick C. K. Hung, Ph.D. is an Associate Professor at the Faculty of Business and Information Technology in University of Ontario Institute of Technology in Canada. Hung has been working with Boeing Research and Technology at Seattle in the USA, and he owns an US patent on "Mobile Network Dynamic Workflow Exception Handling System" with Boeing. In addition, he is a Visiting Researcher at the University of Milan in Italy and the University of Aizu at Fukushima in Japan. He is also an Adjunct Professor at the State Key Laboratory of Software Engineering at Wuhan University in China. He has been an Adjunct Faculty Member at Department of Electrical and Computer Engineering in University of Waterloo in Canada, a Guest Professor at Institute of Computer Science in University of Innsbruck in Austria and Department of Information Engineering and Computer Science in University of Trento in Italy. Before that, he was a Research Scientist with Commonwealth Scientific and Industrial Research Organization (CSIRO) for a health informatics network at Canberra in Australia. He also has prior industrial experience in e-business projects in the North America and Hong Kong. He is a founding committee member of the IEEE International Conference of Web Services, IEEE International Conference on Services Computing, IEEE BigData Congress and IEEE Congress on Services. He is an associate editor of the IEEE Transactions on Services Computing, International Journal of Web Services Research and International Journal of Business Process and Integration Management. His research interests include services computing, business process management, security and privacy. Contact him at patrick.hung@uoit.ca. 